\title{
Topological Study on the Design of Soft Strain Sensors for Simultaneous Multi-point Contact Localization
}

\author{
Leone Costi ${ }^{1}$, Thomas George Thuruthel ${ }^{1}$, Fumiya Iida ${ }^{1}$
}

\begin{abstract}
Soft strain sensors have been widely used for the development of electronic skins both for robotic and wearable applications. To sense contact location on a wide surface, the standard methodology consists of square grids of strain fibers that are able to detect single contact points but fail to detect multiple ones simultaneously. To avoid such a problem, state-ofthe-art technologies implement sequential sampling that isolates each sensing node, but at the cost of a lower sampling rate. This theoretical study proposes a design methodology for multitouch detection for parallel processed grid-based strain sensors. The fundamental idea is to add diagonal grids of varying orientations on top of the standard architecture to achieve multi-touch detection. The maximum number of detectable points and the number of required strain fibers and the overall geometry of the sensor are studied along with the error introduced when trying to sense more contact points than designed for. Overall, compared with state-of-the-art design methodologies, our work provides a guideline for more efficient grid-based architectures that are able to simultaneously detect up to a fixed finite number of contact points.
\end{abstract}

\section{INTRODUCTION}

Advancements in soft strain sensing technologies are vital for the development of delicate and fully conformable electronic skins for robotic and wearable applications [1], [2]. With appropriate design, placement and processing, these sensors can be combined to obtain various multi-modal tactile information such as deformation, force, pressure, and contact location [3], [4]. This work presents a design study on the use of soft strain sensors for simultaneous multipoint contact localization. Similar design studies have been performed for proprioception [5], [6] and robust single-point detection [7], however, not for multi-point contact detection.

There are numerous combinations of design and technologies that be used to develop soft robotic skins for contact localization, each one with its own trade-offs and constraints. All multi-touch sensory arrays can be divided into two based on their sampling process: sequential and parallel. Sequential sensory arrays measure a subset of the sensors at a time, leading to a slower sampling period, but allow decoupling of sensory inter-dependencies, making them highly compact with fewer electrodes. This approach is widely used in current touchscreen sensors as sampling can be performed at very high rates for rigid tactile sensors [8]. Sequential sampling methods can be extended for soft strain sensors too, however, due to typical higher electrical resistance, they will have a much lower sampling rate and a higher chance of signal cross-contamination. Anisotropic electrical impedance

\footnotetext{
${ }^{1}$ The Bio-Inspired Robotics Lab, Department of Engineering, University of Cambridge, UK.
}

tomography with conductive elastomers, capacitive soft sensors [9] and force-sensitive film-based sensors [10], [11] [12], [13] are just a few examples.

Parallel sensory arrays measure all the sensing elements simultaneously, leading to a faster sampling rate. However, the number of simultaneously detectable contacts decreases for the same number of fibers. Examples would be grid sensors made with soft strain sensors [7], [14] or visionbased soft sensors [15], [16]. Based on the applications, the data processing method and the technologies involved must be selected. In this study, we focus on sensory arrays composed of soft strain sensors that are parallelly measured. Such technologies are relatively easier to manufacture, provide higher conformability and faster sampling rate [17]. However, current sensor designs are limited to single-point contact only. This work investigates design rules for extending these technologies for multi-point contact localization.

\section{THEORY}

The fundamental idea of this study is to add diagonal grids of variable orientation on top of the standard architecture to achieve multi-touch detection. (see Figure 1).

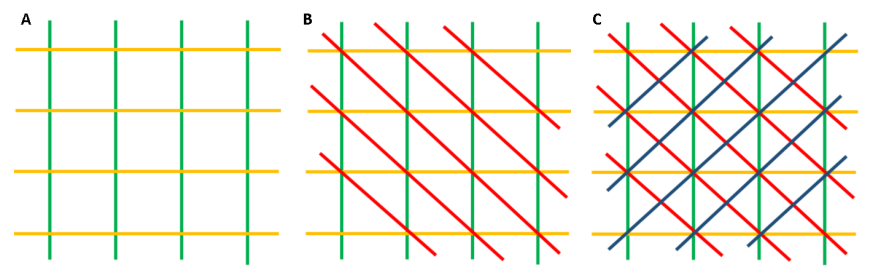

Fig. 1. Grid-based architecture designed to detect (A) one, (B) two and (C) three points simultaneously. Note that each architecture can be manufactured by placing strain fibers parallel to each other after rotating the base structure.

Any grid-based sensor is composed of a set of nodes and a specific unit cell. The latter is the smallest repeatable single unit, while a node is any point in which the horizontal and the vertical piezo-resistive fibers cross each other. Note that, since diagonal fibers are not considered in the definition of nodes, the number of both unit cells and nodes are not affected by the addition of any number of them, but their morphology is. For example, in Figure 1, the full-scale sensor is composed of 9 square unit cells and 16 nodes in all cases. The size of a sensor can be expressed by $N$ and $M$, where the former is the number of horizontal fibers and the latter is the number of vertical fibers. Hence, a sensor will have $N M$ nodes and $(N-1)(M-1)$ unit cells.

For our analysis, we assume that any contact would cover one node: if the touched surface of the sensor contains $p$ 
nodes, it is considered as $p$ distinct contact points. At any moment during the design, diagonals to a rectangle of size $R \times C$ can be added, changing the morphology of the nodes and the unit cells (see Figure 2). $R$ and $C$ are the numbers of unit cells that constitute the length and the width of the rectangle, respectively. For each combination of $R$ and $C$ it is also possible to add the anti-diagonal: the anti-diagonal is the diagonal that goes from the top right corner of the rectangle to the bottom left (i. e. the blue fibers in Figure 1), instead of from top left to bottom right.
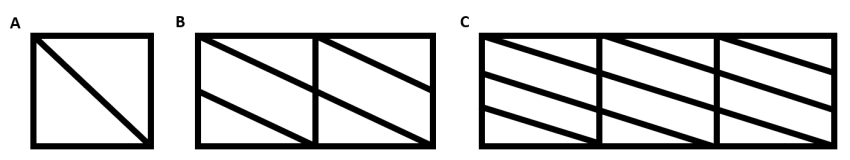

Fig. 2. Examples of (A) $1 \times 1$, (B) $1 \times 2$, and (C) $1 \times 3$ diagonals.

Adding a diagonal increases the number of the fibers in the sensor by $(N-1) C+(M-1) R-1$ and the default square grid has $N+M$ fibers, so the total number of fibers needed to develop a $M \times N$ sensor with $D$ diagonals, denoted by $W$, can be expressed as follows:

$$
W=M+N+\sum_{k=1}^{D}\left((N-1) C_{k}+(M-1) R_{k}\right)-D
$$

Next, new metrics to analyze the performance of a given architecture are introduced: the limit grid and the maximum amount of simultaneously detectable points, $P_{\max }$. The latter can be computed by finding at least 2 sets of nodes that would give the same fibers' output when touched: $P_{\max }$ is then defined as one less than the number of elements in either one of the two sets because the two sets themselves cannot be uniquely identified. As an example, Figure 3 , on the left, illustrates two sets of nodes $\left(A_{\text {old }}\right.$, red dots, and $B_{\text {old }}$, red circles) that satisfy such a requirement, hence, since every set contains 3 elements, this architecture has $P_{\max }=2$. If the sets of a given architecture are known and a diagonal is added to it, the new $A_{n e w}$ and $B_{\text {new }}$ can be calculated following 3 simple steps: candidate selection, symmetric difference, and assignment. Firstly, upon the addition of a diagonal $R \times C$, new candidates nodes need to be selected: a node becomes a candidate if it is located $R$ rows below and $C$ columns to the right of any node belonging to $A_{\text {old }}$ or $B_{\text {old }}$. If an anti-diagonal has been added, the new candidates will be $C$ columns to the left, instead of the right. Next, all the common nodes between the old sets and the new candidates are discarded by taking the symmetric difference. Finally, all the remaining nodes are assigned to $A_{\text {new }}$ and $B_{\text {new }}$ as follows: if the node was previously belonging to $B_{\text {old }}$ or was a candidate with respect to a node in $A_{\text {old }}$, it is added to $B_{\text {new }}$, and if it was previously belonging to $A_{\text {old }}$ or was a candidate with respect to a node in $B_{\text {old }}$, it is added to $A_{\text {new }}$. Figure 3 shows the procedure for the $1 \times 1$ anti-diagonal: the red dots and red circles are the initial $A_{\text {old }}$ and $B_{\text {old }}$, the yellow nodes are the candidates. On the right, nodes are denoted with dots if they belong to $A_{n e w}$ and circles if they belong to $B_{n e w}$. The two nodes in the middle of the grid are discarded while taking the symmetric difference. Therefore $P_{\text {max }}=3$, one less than the elements in $A_{\text {new }}$ or $B_{\text {new }}$.
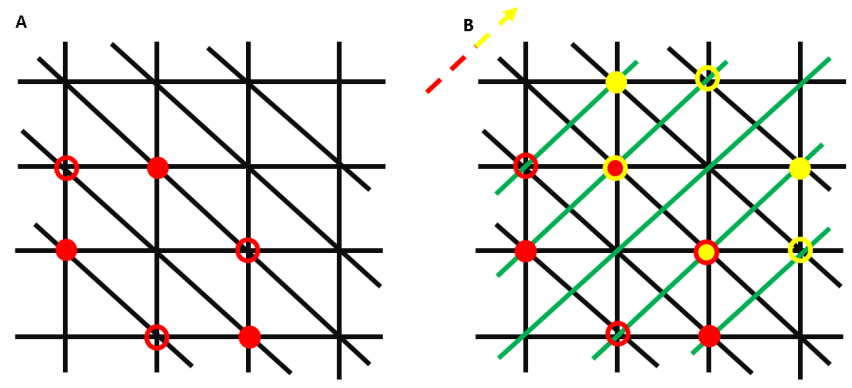

Fig. 3. (A) $1 \times 1$ diagonal architecture with $A_{\text {old }}$ as red dots and $B_{\text {old }}$ as red circles. (B) $1 \times 1$ diagonal and anti-diagonal architecture with $A_{n e w}$ as dots and $B_{\text {new }}$ as circles.

The only exception occurs for the first diagonal that is added to the default square grid: in this case, it is possible to identify two different configurations with a different maximum number of points: one using the previously explained technique, while the other is obtained by considering the vertices of a hexagon inscribed inside a rectangle of dimension $2 R \times 2 C$, as shown on the left of Figure 3 . Note that this second approach is only valid for the first diagonal and it can be used only if $N>2 R$ and $M>2 C$, but it leads to a smaller $P_{\max }$, hence its usage should be preferred every time the grid's dimensions allow it.

Next, it can be noticed that, if the sensor's grid is too small to allow the aforementioned candidate selection, $A_{\text {new }}$ and $B_{\text {new }}$ cannot be defined, leading to the detection up to all the grid's nodes, simultaneously. The limit grid, constituted by $N_{l i m}$ horizontal and $M_{l i m}$ vertical fibers, is the smallest grid that fails to detect contact up to all nodes simultaneously. Since it depends on the number and the characteristics of the diagonals, it can be calculated as follows:

$$
\left\{\begin{array}{l}
N_{l i m}=1+\sum_{k=1}^{D} R_{k} \\
M_{l i m}=1+\sum_{k=1}^{D} C_{k}
\end{array}\right.
$$

Where $D$, as previously stated, is the number of diagonals.

To find the optimal theoretic solution, let's suppose to have a grid $N \times M$, with $N M$ nodes and $W$ fibers. Now a connectivity matrix $T$ of dimensions $W \times N M$ can be built such that $T_{i j}=1$ if the fiber $i$ goes through node $j$ and $T_{i j}=0$ otherwise, according to our hypothesis. Thanks to this matrix it is possible to write the relation between the touched nodes and the output of the sensor as follows:

$$
y=T x
$$

Where $x$ is a vector $N M \times 1$ so that $x_{i}=1$ if the node is touched and $x_{i}=0$ if not and $y$ is the $W \times 1$ output vector. Finally, it is clear that to retrieve $x$ from $y$ the matrix $T$ needs to be of rank $N M$, to avoid multiple valid solutions, therefore the number of fibers needed in a sensor to detect simultaneously all the nodes of the sensor cannot be lower 
than the number of nodes itself $(W \geq M N)$ : this design will be referred as one-wire-per-node in the following discussion.

\section{RESULTS}

The first study that can be performed with our proposed method is how the number of piezo-resistive fibers scales with the size of the sensor. If only square grids are considered (i.e $M=N$ ), different architectures can be designed to detect multiple contact points. Figure 4 shows how the different architecture's number of fibers scale with respect to the size of a square grid $N \times N$.

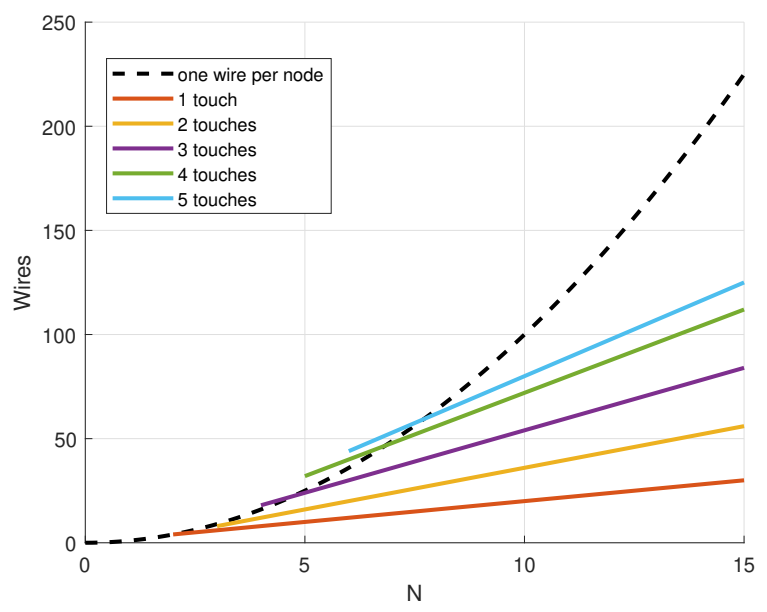

Fig. 4. Number of fibers as a function of dimensions of square grids.

The results for different $P_{\max }$ are compared with the one-wire-per-node approach, able to detect up to all nodes simultaneously: the grid-based approach for a finite number of touches will eventually need fewer fibers than the standard approach since it scales in the order of $N$ instead of $N^{2}$.

When trying to detect a number of points $P>P_{\max }$ some errors are introduced. To estimate the quantitative value of these errors, we can calculate all the combinations of points that cannot be correctly detected. From the previous Section, it is known that any $N_{\text {lim }} \times M_{\text {lim }}$ sub-grid of the grid is going to have two different sets with the same output (see Figure 3), leading to an absolute error of 1 when detecting $P_{\text {max }}+1$ points. Accounting for all the possible $N_{\text {lim }} \times M_{\text {lim }}$ sub-grids in a $N \times M$ grid, the absolute error in detecting $P_{\max }+1$ points is $\left(N-N_{\text {lim }}+1\right)\left(M-M_{\text {lim }}+1\right)$. For $P>P_{\max }+1$, it is sufficient to count all the combinations that contain the $P_{\max }+1$ combinations previously found. That is possible using combinatorial calculus as follows:

$$
e_{a b s}=\left(N-N_{l i m}+1\right)\left(M-M_{l i m}+1\right) \sum_{k=0}^{t} \frac{(\alpha) !}{k !(\alpha-k) !}
$$

Where $\alpha=N M-2\left(P_{\max }+1\right)$ and $t=P-P_{\max }-1$.

The relative error can then be computed dividing the $e_{a b s}$ by all the possible combinations of nodes up to $P$ points, as follows:

$$
e_{r e l}=\frac{e_{a b s}}{\sum_{k=1}^{P} \frac{(N M) !}{k !(N M-k) !}}
$$

Figure 5 shows the relative error of square grids for different values of $N$ in the case of a $1 \times 1$ diagonal and anti-diagonal architecture.

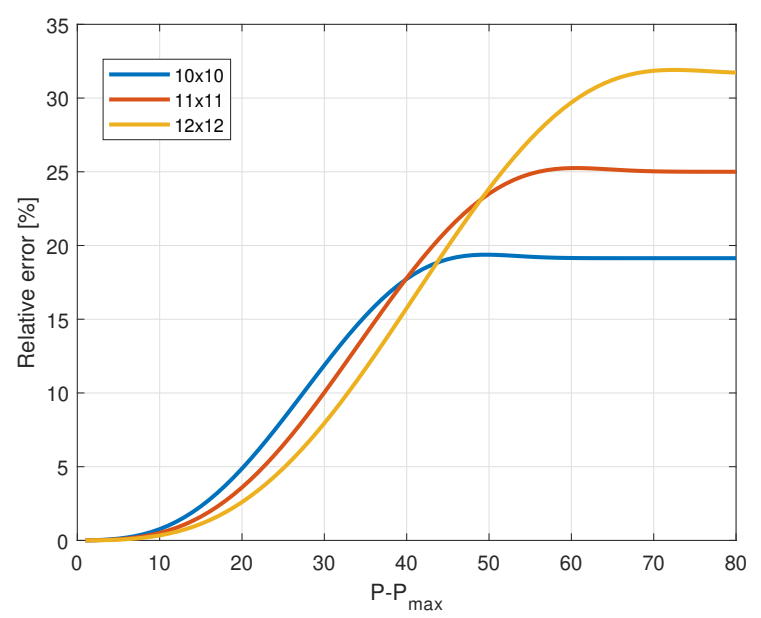

Fig. 5. Relative error of a 1x1 diagonal and anti-diagonal grid as a function of simultaneous detected touches for $P>P_{\max }$.

At first the relative error increases as the number of touched points $P$ increases, since the number of undetectable combinations increases every time a new point is added. Then, the error stabilizes because the number of all possible combinations out-scales the number of wrong ones, leading the trend to stop increasing. The asymptotic value and the increasing rate are strongly dependent on the size of the grid.

\section{CONCLUSION}

This work proposes a design methodology for multi-touch detection for parallel processed grid-based strain sensors. As our contact detection and localization are based on binary information upon contact, any soft strain sensor can be used for physical implementation, irrespective of nonlinearities in their behavior. Our theoretical results provide design guidelines for fabricating these sensory arrays based on the required dimensions and error tolerances. Additional information obtained from the sensor can also be used for estimating the forces applied along with the contact location, provided the sensor data can be modeled appropriately. The elastomeric matrix that would host the fibers has no specific requirements to be met. Hence, the physical implementation could be realized either by silicone-casting the elastomeric matrix in a mold designed to host and keep in position the piezo-resistive fibers or by $3 \mathrm{D}$ printing both matrix and fibers together, depending on the mechanical properties of the selected materials.

\section{ACKNOWLEDGMENT}

This work was supported by the SHERO project, a Future and Emerging Technologies (FET) program of the European Commission (grant agreement ID 828818), and the SMART project, European Union's Horizon 2020 research and innovation under the Marie Sklodowska-Curie (grant agreement ID 860108). 


\section{REFERENCES}

[1] B. Shih, D. Shah, J. Li, T. G. Thuruthel, Y.-L. Park, F. Iida, Z. Bao, R. Kramer-Bottiglio, and M. T. Tolley, "Electronic skins and machine learning for intelligent soft robots," Science Robotics, vol. 5, no. 41, 2020.

[2] D. Chen and Q. Pei, "Electronic muscles and skins: a review of soft sensors and actuators," Chemical reviews, vol. 117, no. 17, pp. 11239 $11268,2017$.

[3] H. Wang, M. Totaro, and L. Beccai, "Toward perceptive soft robots: Progress and challenges," Advanced Science, vol. 5, no. 9, p. 1800541, 2018.

[4] T. G. Thuruthel, B. Shih, C. Laschi, and M. T. Tolley, "Soft robot perception using embedded soft sensors and recurrent neural networks," Science Robotics, vol. 4, no. 26, 2019.

[5] J. Tapia, E. Knoop, M. Mutnỳ, M. A. Otaduy, and M. Bächer, "Makesense: Automated sensor design for proprioceptive soft robots," Soft robotics, vol. 7, no. 3, pp. 332-345, 2020.

[6] U. Culha, S. G. Nurzaman, F. Clemens, and F. Iida, "Svas3: strain vector aided sensorization of soft structures," Sensors, vol. 14, no. 7, pp. 12 748-12770, 2014.

[7] T. G. Thuruthel, J. Hughes, and F. Iida, "Joint entropy-based morphology optimization of soft strain sensor networks for functional robustness," IEEE Sensors Journal, vol. 20, no. 18, pp. 10 801-10810, 2020.

[8] M. Solution@2011, "Multitouch technologies," http: //multi-touch-solution.com/knowledge-base-en 2020.

[9] H. Shi, M. Al-Rubaiai, C. M. Holbrook, J. Miao, T. Pinto, C. Wang, and X. Tan, "Screen-printed soft capacitive sensors for spatial mapping of both positive and negative pressures," Advanced Functional Materials, vol. 29, no. 23, p. 1809116, 2019.

[10] S. Sundaram, P. Kellnhofer, Y. Li, J.-Y. Zhu, A. Torralba, and W. Matusik, "Learning the signatures of the human grasp using a scalable tactile glove," Nature, vol. 569, no. 7758, pp. 698-702, 2019.

[11] L. Pan, A. Chortos, G. Yu, Y. Wang, S. Isaacson, R. Allen, Y. Shi, R. Dauskardt, and Z. Bao, "An ultra-sensitive resistive pressure sensor based on hollow-sphere microstructure induced elasticity in conducting polymer film," Nature communications, vol. 5, no. 1, pp. 1-8, 2014.

[12] H. Lee, D. Kwon, H. Cho, I. Park, and J. Kim, "Soft nanocomposite based multi-point, multi-directional strain mapping sensor using anisotropic electrical impedance tomography," Scientific reports, vol. 7, no. 1, pp. 1-10, 2017.

[13] C. Larson, J. Spjut, R. Knepper, and R. Shepherd, "A deformable interface for human touch recognition using stretchable carbon nanotube dielectric elastomer sensors and deep neural networks," Soft robotics, vol. 6, no. 5, pp. 611-620, 2019.

[14] J. Hughes and F. Iida, "Tactile sensing applied to the universal gripper using conductive thermoplastic elastomer," Soft robotics, vol. 5, no. 5, pp. 512-526, 2018.

[15] R. Li, R. Platt, W. Yuan, A. ten Pas, N. Roscup, M. A. Srinivasan, and E. Adelson, "Localization and manipulation of small parts using gelsight tactile sensing," in 2014 IEEE/RSJ International Conference on Intelligent Robots and Systems. IEEE, 2014, pp. 3988-3993.

[16] A. Yamaguchi and C. G. Atkeson, "Combining finger vision and optical tactile sensing: Reducing and handling errors while cutting vegetables," in 2016 IEEE-RAS 16th International Conference on Humanoid Robots (Humanoids). IEEE, 2016, pp. 1045-1051.

[17] M. Amjadi, K.-U. Kyung, I. Park, and M. Sitti, "Stretchable, skinmountable, and wearable strain sensors and their potential applications: a review," Advanced Functional Materials, vol. 26, no. 11, pp. 16781698, 2016. 